\title{
Postoperative renal impairment is associated with increased length of stay for incisional hernia repair after liver transplantation is
}

\section{James R. Butler ${ }^{1}$, Daniel C. O'Brien ${ }^{1}$, Joshua K. Kays, Kyle Ridlen, Chandrashekhar A. Kubal, Burcin Ekser, Lava Timsina, Jonathan A. Fridell, Richard S. Mangus, John A. Powelson*}

Department of Surgery, Indiana University School of Medicine, Indianapolis, IN, USA

\section{A R T I C L E I N F O}

\section{Article history:}

Received 6 April 2019

Received in revised form 4 June 2019

Accepted 6 June 2019

Available online 29 June 2019

\begin{abstract}
A B S T R A C T
Background: Incisional hernia repair is the most common procedure after orthotopic liver transplantation. Although enhanced recovery protocols are increasingly employed, the post-orthotopic liver transplantation patient may not benefit from all aspects of these models. The aim of the present study is to assess which perioperative interventions and patient factors affect hospital length of stay in a cohort of post-orthotopic liver transplantation patients undergoing incisional hernia repair.

Methods: We conducted a retrospective review of a series of adult patients undergoing incisional hernia repair after orthotopic liver transplantation. The primary endpoint was length of stay. Results were stratified by demographic, intraoperative, and postoperative variables.

Results: Eleven percent (172/1523) of patients who received orthotopic liver transplantation during the study period underwent subsequent incisional hernia repair. Median length of stay was 5 days (range 2-50). The strongest predictor of length of stay was postoperative renal function. Despite liberal intraoperative administration of volume (median $642 \mathrm{~mL} / \mathrm{h}$ ) and brisk intraoperative urine output (median $72 \mathrm{~mL} / \mathrm{h}$ ), postoperative acute kidney injury occurred in $48 \%$ of patients. Those that developed acute kidney injury received less intraoperative volume ( $6 \mathrm{vs} 8.5 \mathrm{~mL} / \mathrm{kg} / \mathrm{h} ; P=.031$ ) and the severity of postoperative renal injury was inversely related to the amount intraoperative volume given.

Conclusions: In patients undergoing incisional hernia repair after orthotopic liver transplantation, postoperative renal function is frequently impaired. Although many aspects of current ERAS protocols may be applied to post-transplant patients, restrictive intraoperative fluid administration strategies should be employed with caution given a high propensity for the development of post-operative acute kidney injury in this complex population.
\end{abstract}

(C) 2019 The Authors. Published by Elsevier Inc. This is an open access article under the CC BY-NC-ND license (http:// creativecommons.org/licenses/by-nc-nd/4.0/).

\section{BACKGROUND}

Incisional hernias $(\mathrm{IH})$ are a common complication following orthotopic liver transplantation (OLT), with rates reported in the literature between 4.9 and 34.3\% [3-19]. As one of the most frequent surgical procedure after OLT, IH repair (IHR), provides a useful model for the evaluation of transplant recipients' response to general surgical operations at large. Although the incidence of IHR after OLT is well described, less attention has been afforded to the perioperative management of this population within the literature.

\footnotetext{
it This Research was presented at the annual meeting of the American Society of Transplant Surgeons, 2-5 June 2018. Seattle, WA

* Corresponding author at: Indiana University School of Medicine, Department of Transplant Surgery, 550 University Bvld. \#4601, Indianapolis, IN 46202. Tel.: +1 317 9444370.

E-mail address: jpowelso@iupui.edu (J.A. Powelson).

1 co-first authors.
}

Enhanced recovery after surgery (ERAS) pathways have recently gained considerable attention across many surgical disciplines. Aimed at reducing the derivative morbidity and cost associated with extended hospital stay, these systems offer standardized guidelines for perioperative management. From their inception in the 1990s, ERAS protocols have been designed in a procedure-specific manner. Within abdominal surgery, the ERAS society has published guidelines for operations ranging from colon resection to pancreaticoduodenectomy. Among other things, these strategies strive to avoid excess fluid administration, and have even adopted the concept of 'permissive oliguria.' [1] Recently, patientspecific variables such as advanced age [2], or emergent presentation, have been found to affect the efficacy of enhanced recovery strategies. As ERAS pathways are currently applied to an increasing variety general surgical procedures, it will be important to consider their effects on unique patient populations within these procedure-specific strategies; unique patient populations deserve unique recovery strategies.

Patients living with a hepatic allograft exhibit unique neurohoromonal stress responses; among other things, these patients exhibit an altered 
Table 1

Predictors of Length of stay for incisional hernia repair after orthotopic liver transplantation.

\begin{tabular}{|c|c|c|c|c|}
\hline Variable & $\begin{array}{l}\text { All } \\
(\mathrm{n}=172)\end{array}$ & $\begin{array}{l}\text { Stay }>5 \text { days } \\
(\mathrm{n}=73)\end{array}$ & $\begin{array}{l}\text { Stay } \leq 5 \text { days } \\
(\mathrm{n}=99)\end{array}$ & $P$ \\
\hline Median time to hernia repair after OLT (d) & 586 & 683 & 538 & .04 \\
\hline Female & $24.4 \%$ & $17.8 \%$ & $29.3 \%$ & .09 \\
\hline Median Age at IHR (y) & 55 & 56 & 53 & .3 \\
\hline Median MELD at OLT & 18 & 18 & 17 & .4 \\
\hline \multicolumn{5}{|l|}{ Technique of Repair } \\
\hline Open Repair & $35.5 \%$ & $43.3 \%$ & $56.7 \%$ & .8 \\
\hline Laparoscopic Repair & $64.5 \%$ & $42.1 \%$ & $57.9 \%$ & \\
\hline Albumin at IHR (mg/dL) & 3.9 & 3.9 & 3.7 & .03 \\
\hline BMI at IHR $\left(\mathrm{kg} / \mathrm{m}^{2}\right)$ & 29.1 & 30.4 & 28.7 & .02 \\
\hline Mesh size $\left(\mathrm{cm}^{2}\right)$ & 702 & 750 & 600 & .1 \\
\hline EBL at IHR $(\mathrm{mL})$ & 30 & 50 & 30 & .1 \\
\hline Length of IHR (min) & 187.5 & 206 & 167 & .005 \\
\hline Epidural anesthesia & $32 \%$ & $42.5 \%$ & $25.3 \%$ & .02 \\
\hline Return of bowel function (d) & 3 & 4 & 3 & .04 \\
\hline \multicolumn{5}{|l|}{ Intraoperative volume measures } \\
\hline \multicolumn{5}{|l|}{ Total volume (mL) } \\
\hline Volume given ( $\mathrm{mL} / \mathrm{min})$ & 10.7 & 10.6 & 10.7 & .4 \\
\hline Intraoperative urine output ( $\mathrm{mL} / \mathrm{min})$ & 1.26 & 1.26 & 1.27 & .1 \\
\hline \multicolumn{5}{|l|}{ Postoperative renal function } \\
\hline Median baseline $\mathrm{Cr}(\mathrm{mg} / \mathrm{dL})$ & 1 & 1 & 1 & .2 \\
\hline Postoperative AKI* & $48.2 \%$ & $71.2 \%$ & $31.3 \%$ & $<.0001$ \\
\hline Median peak $\mathrm{Cr}$ (mg/dL) & 1.3 & 1.9 & 1.3 & .0005 \\
\hline Rise in $\mathrm{Cr}$ & $0.3 \%$ & 0.9 & 0.27 & $<.0001$ \\
\hline \multicolumn{5}{|l|}{ Postoperative liver function } \\
\hline Baseline bilirubin (mg/dL) & 0.8 & 0.7 & 0.8 & .06 \\
\hline Peak bilirubin (mg/dL) & 1.5 & 1.6 & 1.5 & .4 \\
\hline$\%$ Rise in bilirubin & $89 \%$ & $110 \%$ & $77.8 \%$ & .02 \\
\hline
\end{tabular}

$C K D$, Chronic renal disease; HTN, hypertension; BMI, body mass index; IHR, incisional hernia repair; EBL, estimated blood loss.

* Postoperative AKI defined as $>0.3 \mathrm{mg} / \mathrm{dL}$ rise in $\mathrm{Cr}$ from baseline within 24 hours of operation.

vascular hemodynamic response, one that is less sensitive to volume resuscitation. Persistent depression of splanchnic vascular resistance, coupled with the nephrotoxicity of enterohepatic circulated immunosuppression agents, may predispose the native kidney to injury. Although there are currently no enhanced recovery protocols designed for patients with hepatic allografts undergoing subsequent surgery, it is likely that many providers will begin utilizing available procedure-specific protocols in this population; within this climate it important to carefully consider the appropriateness of minimizing perioperative vascular preload.

The aim of the present study is to assess the effect of baseline demographic, and perioperative variables on morbidity and hospital length of stay in patients who have undergone IHR after OLT. Precedent to the anticipated implementation of enhanced recovery protocols in this population, specific attention was afforded to perioperative renal function, as renal injury has well described implications to both graft and patient survival after OLT.

Table 2

Multivariable logistic regression of variables predicting increased length of hospital stay

\begin{tabular}{llll}
\hline Variable & Odds ratio & $95 \% \mathrm{CI}$ & $P$ \\
\hline Female & 0.718 & $0.241-2.14$ & .553 \\
MELD at OLT & 0.972 & $0.892-1.056$ & .501 \\
Laparoscopic Repair & 0.916 & $0.331-2.536$ & .867 \\
Preoperative Albumin & 1.755 & $0.749-2.536$ & .201 \\
BMI & 1.054 & $0.961-1.156$ & .258 \\
Mesh size & 0.999 & $0.998-1.001$ & .763 \\
EBL at IHR & 1.001 & $0.997-1.005$ & .433 \\
Length of Case (min) & 1.004 & $0.997-1.012$ & .224 \\
Epidural & 3.191 & $1.100-9.252$ &. $\mathbf{0 3 3}$ \\
Days to bowel function & 3.373 & $2.017-5.639$ & $\mathbf{. 0 0 1}$ \\
Intraoperative urine output (mL/min) & 0.799 & $0.515-1.238$ & .421 \\
$\quad$ Baseline Serum Cr & 0.755 & $0.221-2.570$ & .653 \\
Postoperative AKI* & 2.786 & $1.129-6.879$ & $\mathbf{. 0 2 6}$ \\
\% rise in Bilirubin & 1.112 & $0.826-1.495$ & .482 \\
\hline
\end{tabular}

* Postoperative Acute Kidney Injury (AKI) defined as $>0.3 \mathrm{mg} / \mathrm{dL}$ rise in $\mathrm{Cr}$ from baseline within 24 hours of operation.

\section{METHODS}

This retrospective study was approved by the Indiana University School of Medicine Institutional Review Board and was Health Insurance Portability and Accountability Act (HIPPA)-compliant. We conducted a review of a prospectively collected database consisting of all patients who underwent OLT between the years of 2005-2016 at our institution. One hundred seventy-two such patients out of 1523 OLTs performed within the same period $(172 / 1523,11 \%)$ were identified as having subsequently undergone IHR, all of whom are included in this analysis. All repairs were performed by a single transplant surgeon who specializes in hernia repair (J.A.P). Both laparoscopic and open repairs were performed utilizing a synthetic mesh underlay approach.

The primary endpoint for this study was hospital length of stay following IHR. Secondary endpoints were the development of postoperative morbidities. Perioperative fluid administered was specifically queried; no patients in this study were enrolled into protocoled volume restriction pathways. Median hospital length of stay post-IHR was 5 days. Patients were stratified into two groups, those who required hospital stays exceeding the 5 day median and those whose length of stay was 5 days or less. Demographic and clinical variables were compared between groups to assess for associations with the primary endpoint. Continuous variables were analyzed using the Wilcoxon-MannWhitney test. Discrete variables were analyzed using the $\chi^{2}$ test.

Multivariable logistic regression of dichotomized length of stay ( $>5$ days versus $</=5$ days) was performed at .05 level of significance. The factors that were included in the model were gender, MELD, laparoscopic versus open procedure, preoperative serum albumin, BMI, mesh size, blood loss, case length, use of epidural, days to return of bowel function, intraoperative urine output, postoperative acute kidney injury, baseline creatinine, and fraction bilirubin rise. Estimated adjusted odds ratio with 95\% confidence intervals were reported. The multivariable analysis was done in Stata/SE 14.2 (Stata Statistical Software: Release 14. College Station, TX).

A secondary logistic regression analysis was conducted to assess for whether or not statistically significant predictors of increased hospital 


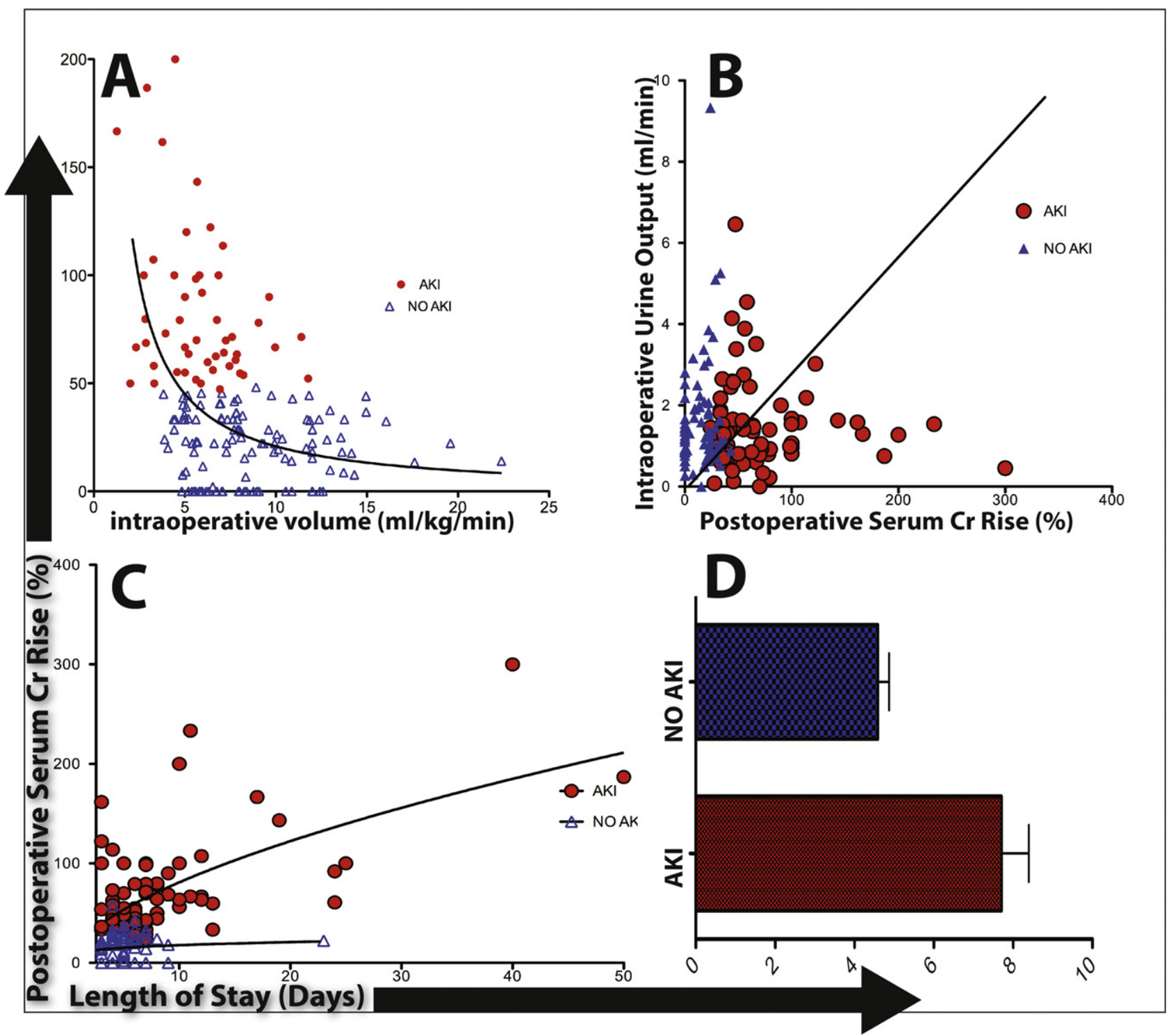

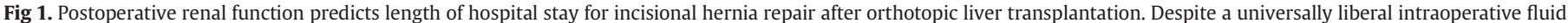

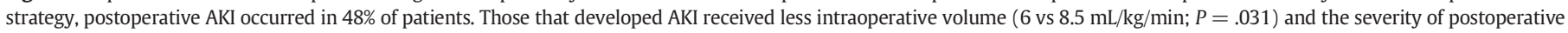

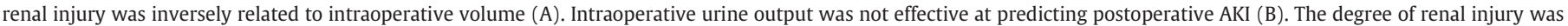
directly related to length of stay (C); patients who developed AKI had a median stay of 8 days compared to 4 days in patients who did not (D).

length of stay were modified by intraoperative therapeutic interventions. Specifically, we sought to determine whether or not postoperative renal impairment was correlated in dependent fashion with volume of crystalloid administered intraoperatively during IHR.

\section{RESULTS}

Eleven percent (172/1523) of patients who received OLT during the years 2005 to 2016 subsequently underwent IHR. Table 1 summarizes demographic and clinical features of the study cohort. The median length of stay was 5 days (range 2-50). Considering an inflection point at $\leq 5$ days hospital stay, there were no statistically significant demographic differences between long stays ( $n=73,42 \%$ ) and short stays ( $n=99,58 \%)$. There was also no difference in hospital stays between those who underwent open versus laparoscopic repairs.

In independent analysis, longer operative times, the use of thoracic epidural catheters, delayed return of bowel function, increased BMI and higher preoperative albumin were found to be associated with increased length of hospital stay. Impaired liver graft function postoperatively, as defined by increased serum bilirubin concentrations, and elevated INR also portended longer hospitalizations. In patients whose lengths of stays exceeded 5 days $(n=73)$, bilirubin increased by an average of $110 \%$ from baseline compared to $78 \%$ in patients with shorter stays $(P=.02)$. Of all collected perioperative variables, postoperative renal function was the most strongly associated with increased length of stay (Table 1 ).

Median baseline creatinine $(\mathrm{Cr})$ was identical between groups $(1 \mathrm{~g} / \mathrm{dL})$. In multivariate analysis, only the development of postoperative acute kidney injury (AKI) (defined as a rise from baseline $\mathrm{Cr}$ concentrations of $>0.3 \mathrm{mg} / \mathrm{dL}$ within 24 hours the IHR), epidural use, and delayed return of bowel function, predicted a longer hospitalization (Table 2). Furthermore, median peak $\mathrm{Cr}$ was significantly higher among patients with longer hospitalizations ( $1.9 \mathrm{vs} 1.3 \mathrm{~g} / \mathrm{dL}, P=.0005$ ).

Median intraoperative fluid administration rates for the cohort were $642 \mathrm{~mL} / \mathrm{h}$. (7 mL/kg/h), and median intraoperative urine output was robust at $72 \mathrm{~mL} / \mathrm{h}$. Despite a universally liberal intraoperative fluid strategy, postoperative AKI occurred in 83 patients $(83 / 172,48 \%)$. Those that developed AKI received less intraoperative volume (6 vs $8.5 \mathrm{~mL} / \mathrm{kg} / \mathrm{h} ; P=.031$ ) and the severity of postoperative renal injury was inversely related to intraoperative volume (Fig 1,A). Intraoperative 
urine output was not effective at predicting postoperative AKI (Fig 1, B). The degree of renal injury was directly related to length of hospital stay (Fig 1, C); patients who developed AKI had a median stay of 8 days compared to 4 days in patients who did not (Fig 1, D).

\section{Discussion}

The utilization of OLT is on the rise, and post-transplant survival continues to improve [20]. With this success, liver transplant patients represent an increasing population requiring non-transplant surgical care. IHR is a common general surgical procedure performed after OLT, and may therefore provide a useful model for the general perioperative management of this cohort. As these procedures are increasingly performed outside of a transplant center, it will be important for all involved practitioners to consider the unique physiology that affects postoperative outcome.

The recent success of ERAS protocols in reducing complications and length of hospital stay make their application attractive to anesthesiologists and surgeons [21]. Of particular relevance to the current study, these protocols focus on judicious intraoperative fluid management. Although from their inception, ERAS protocols recommend "goal directed fluid management strategies" [22], there is little consensus as to what constitutes appropriate goals or effective monitoring methods to assess these goals [23]. In major abdominal surgeries or in the emergent setting, invasive hemodynamic monitoring apparatuses (e.g., arterial line, central venous or pulmonary artery pressure measurements, serial arterial blood gasses) aid assessment of volume status and fluid responsiveness. However, such monitors are seldom used in routine elective surgery, forcing practitioners to rely instead on predetermined volume targets, urine output, and non-invasive pressure monitors to gauge intraoperative circulating volume [24]. In this scenario, a consensus statement from the international enhanced recovery partnership champions limiting intraoperative fluid administration to $2 \mathrm{~mL} / \mathrm{kg} / \mathrm{h}$. [25] Although this may be applicable to a general surgical population, such an approach may prove harmful to patients with existing hepatic allografts and deserves careful consideration.

Successful perioperative management of transplant patients requires strict attention to allograft function. As shown within the present dataset, some degree of allograft dysfunction is common after IHR. Even moderate degrees of hepatic dysfunction can affect renal perfusion through systemically altered vascular response [26]; decreased hepatic metabolism causes a surplus of vasodilatory nitric oxide and endothelial factors that decrease systemic vascular resistance and activate the renin-angiotensin system $[27,28]$. Exacerbating this effect is a derivative increase in splanchnic resistance. While typical surgical patients may tolerate up to a $30 \%$ decrease in blood pressure without changes to systemic blood pressure or heart rate, the splanchnic -and graft- perfusion may be compromised with as little as $10 \%$ volume depletion. Therefore, this marginal deficit escapes even stringent parameters of a goal directed fluid strategy.

Potententiating the effects of excess systemic vasodiliatory mediators on renal perfusion is a patency of portosystemic collateral vessels which are known to persist long after liver transplantation [29]. The resultant splanchnic hyperkinetic circulation, coupled with the residual low superior mesenteric artery (SMA) impedance after liver transplant [30], can decrease renal perfusion despite a normal cardiac output. Aside from challenging the conventional measurements of goal directed resuscitation, these regional perfusion aberrations create a potentially double edged sword: activation of the renin-angiotensin system while simultaneously shunting the effects of volume towards postoperative ileus through the SMA, which itself may potentate nephrotoxicity of conventional immunosuppressive agents that are enterohepaticly circulated; daily monitoring of immunosuppression levels is paramount.

Limitations of the current study are (i) retrospective, (ii) single center, (iii) and single-surgeon. However, these limitations may be relegated by the large sample size; to date this represents the largest series of hernia repairs after OLT. Furthermore, uniformity of operative technique and postoperative management throughout this series likely highlights effects of non-procedural perioperative variables, such as renal function and intraoperative volume administration. Although a randomized approach to the study of recovery pathways for this population would be ideal, it is our contention that the present data -although retrospective- should caution the application of perioperative fluid restriction.

This study, which studied a large cohort of OLTs, demonstrates that post-operative renal impairment is common in IHR after OLT. Likely a multifactorial process, it is important to recognize that this occurred even with a 'liberal' intraoperative volume strategy, and meticulous postoperative immunosuppression adjustment. Furthermore, greater intraoperative volume administration was associated with decreased incidence of postoperative AKI, without extending length of hospital stay within this population.

These findings have several important implications. First, it suggests that traditional endpoints guiding intraoperative fluid administration may not be useful in preventing kidney injury in OLT recipients undergoing subsequent general surgical operations. Second, since even a fairly liberal fluid administration strategy did not protect against AKI development, fluid restrictive strategies such as those advocated in ERAS protocols may subject this unique population to increased risk for renal impairment. Though such protocols have been shown to decrease length of hospital stay in select populations, this effect is likely to be mitigated in OLT patients undergoing subsequent surgeries given their high susceptibility to kidney injury.

In conclusion, patients undergoing IHR after OLT, postoperative renal function is frequently impaired independent of intraoperative venous preload. Potentially owed to the enterohepatic circulation of nephrotoxic immunosuppression, this suggests an important perioperative consideration for OLT patients undergoing general surgery procedures. Although many aspects of current ERAS protocols may be applied to post-transplant patients, restrictive intraoperative fluid administration strategies should be employed with caution given a high propensity for the development of post-operative AKI in this complex population. Although prospective randomized assessment of ERAS protocols in patients with a hepatic allograft will be necessary, the current data suggests that perioperative fluid restriction may not be an appropriate component of such strategies.

\section{AUTHOR CONTRIBUTION}

All authors contributed to study designs and review as indicated (Butler, concept, data collection, data analysis, draft, revision; O'Brien, data collection, analysis, drafting, revision; Kays, data analysis, revision; Ridlen, data collection, revision, Kubal, analysis, revision; Timsinaanalysis, revision; Ekser, analysis, revision; Fridell, analysis, revision; Mangus, analysis, revision Powelson, concept, analysis, drafting revision).

All authors have given final approval to the manuscript as submitted.

\section{CONFLICT OF INTEREST}

No known conflicts of interest are associated with this publication.

\section{FUNDING SOURCES}

there has been no significant financial support for this work that could have influenced its outcome.

\section{References}

1 Ayvazoglu Soy EH, Kirnap M, Yildirim S, Moray G, Haberal M. Incisional Hernia After Liver Transplant. Exp Clin Transplant 2017;15(Suppl 1):185-9. 
2 de Goede B, Eker HH, Klitsie PJ, van Kempen BJ, Polak WG, Hop WC, et al. Incisiona hernia after liver transplantation: risk factors and health-related quality of life. Clin Transplant 2014;28(7):829-36.

3 Fikatas P, Schoening W, Lee JE, Chopra SS, Seehofer D, Guckelberger O, et al. Incidence, risk factors and management of incisional hernia in a high volume liver transplant center. Ann Transplant 2013;18:223-30.

4 Gomez R, Hidalgo M, Marques E, Marin L, Loinaz C, Gonzalez I, et al. Incidence and predisposing factors for incisional hernia in patients with liver transplantation. Hernia 2001;5(4):172-6.

5 Janssen H, Lange R, Erhard J, Malago M, Eigler FW, Broelsch CE. Causative factors, surgical treatment and outcome of incisional hernia after liver transplantation. Br J Surg 2002;89(8):1049-54

6 Kahn J, Muller H, Iberer F, Kniepeiss D, Duller D, Rehak P, et al. Incisional hernia following liver transplantation: incidence and predisposing factors. Clin Transplant 2007;21(3):423-6.

7 Kurmann A, Beldi G, Vorburger SA, Seiler CA, Candinas D. Laparoscopic incisional hernia repair is feasible and safe after liver transplantation. Surg Endosc 2010;24(6): 1451-5.

8 Montalti R, Mimmo A, Rompianesi G, Serra V, Cautero N, Ballarin R, et al. Early use of mammalian target of rapamycin inhibitors is an independent risk factor for incisional hernia development after liver transplantation. Liver Transpl 2012;18(2):188-94.

9 Muller V, Lehner M, Klein P, Hohenberger W, Ott R. Incisional hernia repair afte orthotopic liver transplantation: a technique employing an inlay/onlay polypropylene mesh. Langenbecks Arch Surg 2003;388(3):167-73.

10 Ozgor D, Dirican A, Ates M, Yilmaz M, Isik B, Yilmaz S. Incisional hernia in recipients of adult to adult living donor liver transplantation. World J Surg 2014;38(8):2122-5.

11 Piardi T, Audet M, Panaro F, Gheza F, Cag M, Portolani N, et al. Incisional hernia repair after liver transplantation: role of the mesh. Transplant Proc 2010;42(4):1244-7.

12 Pillebout E, Nochy D, Hill G, Conti F, Antoine C, Calmus Y, et al. Renal histopathological lesions after orthotopic liver transplantation (OLT). Am J Transplant 2005;5(5): 1120-9

13 Shi LW, Verran D, Rao AR, Stewart GJ, McCaughan GW. Incisional hernia following orthotopic liver transplantation. Transplant Proc 2003;35(1):425-6.

14 Smith CT, Katz MG, Foley D, Welch B, Leverson GE, Funk LM, et al. Incidence and risk factors of incisional hernia formation following abdominal organ transplantation. Surg Endosc 2015;29(2):398-404.

15 Sommacale D, Nagarajan G, Lhuaire M, Dondero F, Pessaux P, Piardi T, et al. Surgica procedures in liver transplant patients: A monocentric retrospective cohort study Int J Surg 2017;41:58-64.

16 Vardanian AJ, Farmer DG, Ghobrial RM, Busuttil RW, Hiatt JR. Incisional hernia after liver transplantation. J Am Coll Surg 2006;203(4):421-5.
17 Vennarecci G, Masciana G, De Werra E, Sandri GB, Ferraro D, Burocchi M, et al. Effectiveness and versatility of biological prosthesis in transplanted patients. World J Transplant 2017;7(1):43-8.

18 Miller TE, Roche AM, Mythen M. Fluid management and goal-directed therapy as an adjunct to Enhanced Recovery After Surgery (ERAS). Can J Anaesth 2015;62(2): 158-68.

19 Forsmo HM, Erichsen C, Rasdal A, Korner H, Pfeffer F. Enhanced Recovery After Colorectal Surgery (ERAS) in Elderly Patients Is Feasible and Achieves Similar Results as in Younger Patients. Gerontol Geriatr Med. 2017;3:2333721417706299.

20 Jain A, Reyes J, Kashyap R, Dodson SF, Demetris AJ, Ruppert K, et al. Long-term survival after liver transplantation in 4,000 consecutive patients at a single center. Ann Surg 2000;232(4):490-500.

21 Gustafsson UO, Scott MJ, Schwenk W, Demartines N, Roulin D, Francis N, et al. Guidelines for perioperative care in elective colonic surgery: Enhanced Recovery After Surgery (ERAS(R)) Society recommendations. Clin Nutr 2012;31(6):783-800.

22 Lassen K, Soop M, Nygren J, Cox PB, Hendry PO, Spies C, et al. Consensus review of optimal perioperative care in colorectal surgery: Enhanced Recovery After Surgery (ERAS) Group recommendations. Arch Surg 2009;144(10):961-9.

23 Navarro LH, Bloomstone JA, Auler Jr JO, Cannesson M, Rocca GD, Gan TJ, et al. Perioperative fluid therapy: a statement from the international Fluid Optimization Group. Perioper Med (Lond) 2015;4:3.

24 Kaye A, Riopelle J, Miller R. Intravascular Fluid and electrolyte physiology. 7 ed. Philidelphia. Churchill Livingstone; 2009.

25 Mythen MG, Swart M, Acheson N, Crawford R, Jones K, Kuper M, et al. Perioperative fluid management: Consensus statement from the enhanced recovery partnership. Perioper Med (Lond) 2012;1:2.

26 Hilmi IA, Damian D, Al-Khafaji A, Planinsic R, Boucek C, Sakai T, et al. Acute kidney injury following orthotopic liver transplantation: incidence, risk factors, and effects on patient and graft outcomes. Br J Anaesth 2015;114(6):919-26.

27 Alexander B. The role of nitric oxide in hepatic metabolism. Nutrition 1998;14(4): 376-90.

28 Hollenberg SM, Waldman B. The Circulatory System in Liver Disease. Crit Care Clin 2016;32(3):331-42.

29 Chezmar JL, Redvanly RD, Nelson RC, Henderson JM. Persistence of portosystemic collaterals and splenomegaly on CT after orthotopic liver transplantation. AJR Am J Roentgenol 1992;159(2):317-20.

30 Piscaglia F, Zironi G, Gaiani S, Mazziotti A, Cavallari A, Gramantieri L, et al. Systemic and splanchnic hemodynamic changes after liver transplantation for cirrhosis: a long-term prospective study. Hepatology 1999;30(1):58-64. 